\title{
Epidemiological and clinical characteristics of Streptococcus pneumoniae infections in a tertiary care centre in Bahrain (2010-2014)
}

Jameela Alsalman ${ }^{1}, K^{\prime}$ hadija Alshehabi ${ }^{1}$, Sara Salah ${ }^{1}$, Fatima Ahmed ${ }^{1}$, Hasanin Khudhair ${ }^{1}$, Sajeda Sabt ${ }^{1}$, Hussain Redha ${ }^{1}$, Muneer Mahdi ${ }^{1}$, Ali Madan ${ }^{1}$, Hawra Kamal ${ }^{2}$, Noor Albalooshi ${ }^{3}$

\section{Abstract}

Objective: To describe the epidemiology of invasive and non-invasive pneumococcal disease during a 5-year period in a major hospital in Bahrain.

Design and Setting: This retrospective study investigates cases of pneumococcal diseases among patient $\geq 14$ years old during a fiveyear period (2010-2014), who attended Salmaniya Medical Complex, Bahrain.

Patients and Methods: Laboratory data were obtained from inpatient and outpatient with cultures positive for Streptococcus pneumoniae. Demographics and clinical records from medical files of patients were then reviewed.

Results: A total of 144 culture positive S. pneumoniae representing 139 patients met the inclusion criteria. Ninety-five patients had noninvasive pneumococcal disease and 44 patients had invasive pneumococcal disease. Pneumococcal disease was more common among male patients (58.99\%) with mean age of 56-year (15-95). The most common comorbidities were hypertension, Diabetes Mellitus followed by cardiovascular disease. Twenty-seven patients received mechanical ventilation during their admission, and 13 patients were admitted to the intensive care unit. The overall mortality rate recorded in our centre was $14.4 \%$.
1 Department of Internal Medicine, Salmaniya Medical Complex, Salmaniya, BAHRAIN

2 School of Postgraduate Studies, Royall College of Surgeons in Ireland.

3 Microbiology Laboratory, Salmaniya Medical Complex, BAHRAIN.

Contact information:

Jameela Alsalman.

Đ jalsalman@hotmail.com 
Conclusion: This study supports the current literature where pneumococcal infection is more prevalent in elderly and those with comorbidities. The overall mortality was low and related to age, certain chronic medical illness, admission to intensive care unit and need for mechanical ventilation.

\section{Keywords}

Streptococcus pneumonia; Pneumococcal Diseases; Epidemiological; Bahrain.

\section{Introduction}

Streptococcus pneumoniae, is a Gram-positive diplococcus, causes a spectrum of diseases; ranging from invasive to non-invasive pneumococcal disease (NIPD) [1]. Invasive pneumococcal disease (IPD) refers to isolation of $S$. pneumoniae from a normaIly sterile body sites like blood, cerebrospinal fluid (CSF) or pleural fluid [2]. IPD can manifest as serious diseases such as bacteremia, meningitis and bacteremic pneumonia. NIPD includes the commonly occurring diseases like sinusitis, acute otitis media and non-bacteremic pneumonia. S. pneumoniae is considered the most common cause of community acquired pneumonia (CAP) [1].

Risk factors for developing pneumococcal disease include age, coexisting medical illness such as chronic obstructive pulmonary disease (COPD), chronic heart disease and Diabetes Mellitus [3] The risk of IPD and sepsis has been reported to be increased among patients older than 65 years old with chronic underlying conditions. S. pneumoniae has been shown to occur more frequently in immunocompromised individuals, and possibly with more frequent relapses [4].

Gram stains and culture are routinely used as the first step to identify S. pneumoniae, while new laboratory methods have been introduced such as antigen and nucleic acid detection assays [5-6]. In the United States alone, 4 million pneumococcal disease episodes occur annually with 22,000 deaths among adults [7]. In addition, over 600,000 cases are admitted each year to the hospitals with pneumococcal pneumonia [8]. S. pneumoniae is responsible for considerable mortality reaching as high as 60\% in elderly patients with pneumococcal bacteremia and $22 \%$ in adults with pneumococcal meningitis [1].

This study aims to provide an overview of the burden of culture-proven pneumococcal disease in patients 14 years of age and older who were admitted to Salmaniya medical complex during 20102014 period. To the best of our knowledge, this is the first report describing the epidemiology of Streptococcus pneumoniae infection in adults of Bahrain. A secondary aim of the present study is to compare our findings to that in other parts of the world.

\section{Methods}

\section{Study design and setting}

The study was conducted as a retrospective, descriptive study of patients 14 years and older with cultures positive for Streptococcus pneumonia between January 2010 and December 2014. This age cut-point was included as it covers patients treated in adult wards in our unit. The study had been conducted in Salmaniya Medical Complex, which 
is the main tertiary care hospital in the Kingdom of Bahrain with around 1200 beds and covering around $80 \%$ of the population in the Kingdom of Bahrain (1.4 million as of 2017).

\section{Data collection and participants}

Data were obtained from Microbiology laboratory electronic database for all patients with positive culture specimens for Streptococcus pneumoniae. Specimens included blood, CSF, pleural fluid, sputum, deep tracheal aspirate (DTA), bronchial wash, eye discharge, middle ear fluid, skin, abscess, wound, vaginal swab, vitreous fluid, and synovial fluid. In our labs, standard microbiological methods are used to identify S. pneumoniae.

Demographics and clinical characteristics were then retrieved from medical files and entered in Excel sheets. Data collected included patient demographics (age, sex), specimen collection site, data of positive culture, admission diagnosis, coexisting medical conditions (Diabetes Mellitus, hypertension, chronic lung disease, chronic liver disease, chronic kidney disease, sickle cell disease, hypothyroidism, stroke, cardiovascular disease), immunocompromising conditions, hematological malignancies, solid tumors, chemotherapy, organ transplant receiver, smoking, length of stay for hospitalized patients, need for mechanical ventilation, admission to intensive care unit (ICU), and mortality. In-hospital mortality was defined as death from any cause during the pneumococcal-related admission [10]. The length of stay (LOS) was calculated as the number of days in the hospital. Case fatality rate (CFR) was calculated by dividing the proportion of persons who die from a specified disease among all individuals diagnosed with the disease over a certain period of time.

\section{Exclusion criteria}

Hospital-acquired infections; defined as infections occurring 48 hours after admission [11].

\section{Definitions}

Invasive pneumococcal disease (IPD) was considered as the isolation of $S$. pneumoniae from sterile body site such as blood, cerebrospinal fluid (CSF), and/ or pleural fluid. Non-invasive pneumococcal disease (NIPD) included non-bacteremic pneumonia cases and those with isolates from non-sterile sites such as middle ear fluid and eye discharge.

\section{Statistical Analysis}

The data was processed and analyzed using Microsoft Excel 2016 and the Statistical Package for the Social Sciences (SPSS), version 25 (IBM Corp., Chicago, Illinois, USA).

\section{Ethical considerations}

All data were dealt with in complete privacy. The study was approved by the research committee of the Ministry of Health in Bahrain.

\section{Results}

\section{Age and gender}

Eighty-two cases (58.99\%) occurred in male patients, while 57 (41.01\%) in female patients. Mean age was 56 years (SD 18.048), ranged from 15 to 95 years of age, with 47 (33.81\%) cases occurring among patients $\geq 65$ years old. Figure 1 illustrates the frequency of IPD and NIPD in relation to age

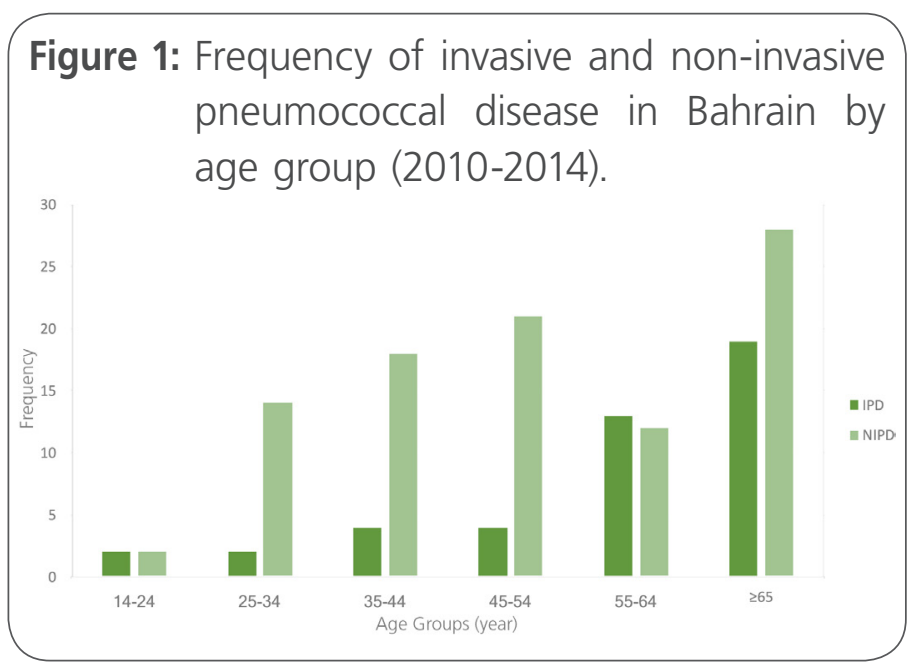


groups. In addition, characteristics of the study cohort are summarized in Table 1.

Table 1. Characteristics and outcomes of the study cohort $(n=139)$.

\begin{tabular}{|l|c|c|}
\hline \multirow{2}{*}{\multicolumn{1}{l|}{ Characteristics }} & \multicolumn{2}{c|}{ All patients } \\
\cline { 2 - 3 } & $\%$ & $\mathrm{n}=139$ \\
\hline Age, year & \multicolumn{2}{|c|}{$56 \pm 18.048$} \\
\hline Mean \pm SD & 47 & 33.81 \\
\hline Patients $\geq 65 y$ & & \\
\hline Gender & 82 & 58.99 \\
\hline Males & 57 & 41.01 \\
\hline Females & 43 & 30.94 \\
\hline Out-patients & 96 & 69.06 \\
\hline In-patients & 27 & 19.42 \\
\hline Mechanical ventilation & 13 & 9.35 \\
\hline ICU & $17 / 118$ & 14.41 \\
\hline Overall Mortality & $101 / 118$ & 85.59 \\
\hline Treated successfully & &
\end{tabular}

\section{Clinical Manifestations}

During the study period 144 positive specimens of S. Pneumonia were recorded in our study. Cases meeting study criteria constituted of 139 patients;
$95(68.35 \%)$ with the non-invasive pneumococcal disease and 44 (31.65\%) with invasive pneumococcal disease. S. pneumococci were isolated from the following sites: DTA or sputum in 62 , blood 43 , CSF in 3, and 36 from other body sites.

\section{Comorbidity}

The most common comorbidities were hypertension, Diabetes Mellitus followed by cardiovascular disease. Moreover, 17 (12.2\%) patients were smokers, 14 (10.1\%) had chronic lung disease, 10 $(7.2 \%)$ had solid tumors, and 7 (5\%) were receiving chemotherapy therapy for their comorbidities as shown in Table 2.

\section{Complications/Outcomes}

Out the total study population, 13 patients were admitted to the intensive care unit (ICU); of those 4 had IPD and 9 had NIPD. Twenty-seven patients needed treatment with mechanical ventilation during their admission; 13 with IPD and 14 with NIPD. Figure 2 illustrates the frequency of complications in both IPD and NIPD groups. The length of stay (LOS) for hospitalized patients, varied between one and 151 days (mean 26.4 days, median 9 days).

Table 2. Co-morbidities in 126 patients with pneumococcal disease; frequency, proportion, Case Fatality Rate(CFR) and relative risk(RR) to die.

\begin{tabular}{|l|c|c|c|c|c|c|c|}
\hline \multicolumn{1}{|c|}{ Co-morbidity } & N & \% & No. died & CFR & RR of death & 95\% Cl & P value \\
\hline Hypertension & 42 & 30.3 & 6 & 15 & 1.0636 & 0.4244 to 2.6658 & 0.895 \\
\hline Diabetes Mellitus & 42 & 30.3 & 5 & 13.2 & 0.8772 & 0.3328 to 2.3121 & 0.790 \\
\hline Cardiovascular diseases & 28 & 20.1 & 7 & 26.9 & 2.4769 & 1.0458 to 5.8665 & 0.040 \\
\hline Chronic lung disease & 14 & 10.1 & 1 & 9 & 0.6080 & 0.0889 to 4.1578 & 0.598 \\
\hline Chronic kidney disease & 12 & 8.6 & 2 & 16.7 & 1.1778 & 0.3056 to 4.5399 & 0.814 \\
\hline Chronic liver disease & 4 & 2.9 & 2 & 50 & 5.1111 & 2.0187 to 12.9407 & 0.009 \\
\hline Stroke & 4 & 2.9 & 2 & 66.7 & 5.1111 & 2.0187 to 12.9407 & 0.009 \\
\hline Sickle cell disease & 6 & 4.3 & 0 & - & - & - & - \\
\hline Hematological malignancy & 3 & 2.2 & 1 & 33.33 & 2.3958 & 0.4539 to 12.6466 & 0.302 \\
\hline Solid tumors & 10 & 7.2 & 4 & 40 & 3.3231 & 1.3317 to 8.2922 & 0.016 \\
\hline Chemotherapy & 7 & 5 & 1 & 14.3 & 0.9911 & 0.1527 to 6.4328 & 0.922 \\
\hline Smoker & 17 & 12.2 & 2 & 11.8 & 0.7922 & 0.1987 to 3.1584 & 0.737 \\
\hline HIV & 0 & - & - & - & - & - & -
\end{tabular}


Vol. 7 No. $2: 3$ doi: $10.3823 / 0808$

Table 3. Complications of pneumococcal disease in 139 patients: frequencies, case fatality rate, relative risk of death.

\begin{tabular}{|l|c|c|c|c|c|c|c|}
\hline \multicolumn{1}{|c|}{ Complication } & Frequency & Discharged successfully & No. died & CFR & RR & $\mathbf{9 5 \%}$ Cl & P value \\
\hline Mechanical ventilation & 27 & 14 & 13 & $48.1 \%$ & 10.95 & $3.89-30.84$ & 0.0001 \\
\hline Admitted to ICU & 13 & 8 & 5 & $38.5 \%$ & 3.37 & $1.41-8.03$ & 0.0111 \\
\hline
\end{tabular}

Figure 2: Frequency of complications and mortality in patients with IPD and NIPD.

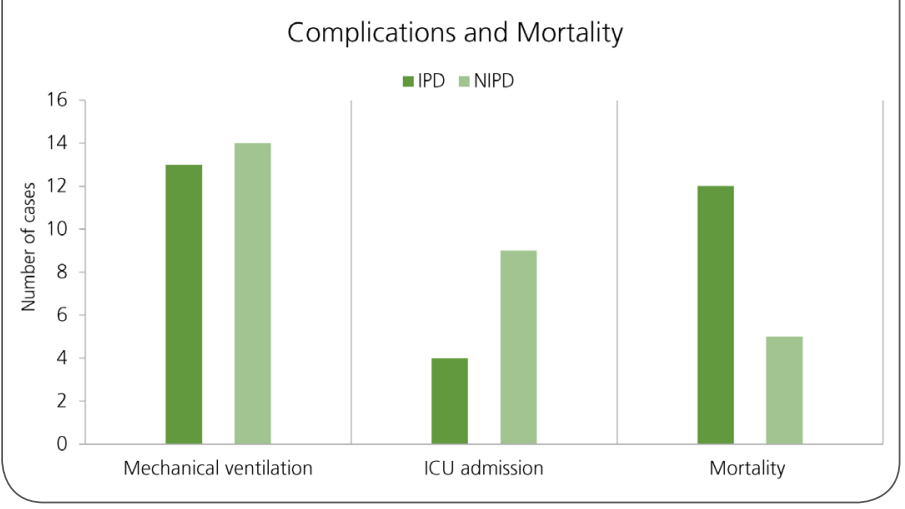

Mortality profile was missing for 21 patients. Out of the remaining 118 patients; 101 (85.59\%) patients were treated successfully and discharged. There were 17 deaths, giving an inpatient mortality rate of $18.28 \%$ in both IPD and NIPD cases, and an overall mortality rate of $14.4 \%$. Twelve of those died had IPD, and had 5 NIPD. Table 3 illustrates the case fatality rate among patients who developed complications.

\section{Discussion}

A previous study has reported the incidence of pneumococcal infections in Bahrain, but it focused exclusively on the paediatric population [9].

Overall, this study has shown that non-invasive pneumococcal disease was the most common presentation among our population. Other investigators in United States [10] and Lebanon [12] have reported similar results.

It is well established in the literature that $S$. pneumoniae have higher incidence in the elderly and among male patients [3]). Older age has not been associated with higher incidence only, but also with more severe disease [13] and higher mortality rates [13-15]. The pattern of age-related incidence and mortality which was noted in our population as well as in terms of gender predominance, males predominated was similar to what has been reported in other countries, such as United States [10], Lebanon [12], and Taiwan [16-17].

The most commonly observed comorbidities in our study were hypertension, Diabetes Mellitus, and chronic cardiovascular disease, however, the mortality rate was not significantly higher in diabetic and hypertensive patients. When compared to findings from other countries. We have noticed a geographical variation in relation to reported comorbidities among patients with pneumococcal disease. In the Gulf Corporation Countries (GCC) diabetes ranked among the top comorbidities [1820], where as much lower rates were reported in Italy [13], Denmark, and the United Kingdom [21]. This may reflect the underlying geographical variations in Diabetes prevalence. GCC countries have the highest prevalence of diabetes in the Middle East and North Africa region with 21\%, 19.6\% and $19.3 \%$ in Saudi Arabia, Bahrain and United Arab Emirates, respectively [22-24]. This is in contrast to Europe where it doesn't exceed $5.1 \%$ in Italy as example [25].

On the other hand, chronic cardiac conditions have been reported in most countries to have high risk of mortality $[17,21,26]$. In the literature, congestive heart failure has been recognized as having significant risk for mortality, while ischemic heart disease has not [3]. In our study, however, all heart diseases were included under one title, so it was 
not possible to make this distinction. Furthermore, in courtiers like the United States where Human Immunodeficiency Virus (HIV) is prevalent, a study reported HIV as the most common underlying conditions among patients with pneumococcal disease and second most common among those who died of pneumococcal disease [27], while no HIV infected case was diagnosed in our study.

Despite that chronic lung diseases are reported to be associated with significant risk for pneumococcal infection [3] and prevalent among investigated cohorts in many studies [19, 19, 28]. This study shows that the proportion of patients with lung disease didn't exceed $10.1 \%$ (14/139) with one death occurring among them, while other medical conditions related to high mortality were solid tumors, which is consistent with the reports of literature $[1,28]$.

The mortality rates we observed agreed with other studies, which range from $6 \%$ to greater than $50 \%$ depending on a number of factors, including age, disease severity and comorbid conditions [10]. The overall in-hospital mortality rate in our study was $18.28 \%$ for both IPD and NIPD cases and the overall mortality rate was $14.4 \%$, which is comparable to rates in US (13.6\%) [10].

Mortality was associated with age, certain comorbidities (cardiovascular disease, chronic liver disease, solid tumors), admission to ICU and the need for mechanical ventilation. Mechanical ventilation was reported as one of the factors associated with a fatal outcome among adult patients with bacteremic pneumococcal in Finland by which a need for mechanical ventilation was the most common complication (16\%) [29]. Whereas. in our study, a higher rate $(19.4 \%$, ) of patients receiving mechanical ventilation. Ricketson et al. [28] have reported that ICU admission, mechanical ventilation and advanced age were found to have a significant contribution in mortality among patients with IPD. Although the presence of comorbidities contributes to high mortality, it is difficult to ascer- tain the attributable risk of pneumococcal disease to mortality in patients admitted to ICU as overall mortality is high in ICU patients [30].

Finally, a number of potential limitations need to be considered. First, this study has a few limitations. As it required cases that were diagnosed primarily by cultures. Prior antibiotic use or failure to collect cultures by treating physicians may underestimate the real burden of the disease. The small sample size may be a limiting factor as well. The relatively small number of positive cultures compared to the number of samples processed per case may be explained by the usual care of these patients, as most of the patients have received several courses of antibiotics in out-patient or in-patient settings prior to admission to this hospital. Moreover, a proper selection of antimicrobial treatment is also important factor for successful control of pneumococcal infection.

\section{Conclusion}

This study provided baseline epidemiological data on invasive and non-invasive pneumococcal disease in a major hospital in Bahrain. It showed the high burden of disease among males and elderly people and those with hypertension, diabetes and cardiovascular diseases. Non-invasive pneumococcal disease was the most frequent diagnosis among our study population. These findings are similar to reports from other GCC countries. The mortality reported in this study would further necessitate the need or improvement of national preventive measure. 


\section{References}

1. Centers for Disease Control and Prevention. Epidemiology and Prevention of Vaccine-Preventable Diseases. Washington D.C: Public Health Foundation; 2015. Available from: https://www. cdc.gov/vaccines/pubs/pinkbook/front-matter.html.

2. Andresen DN, Collignon PJ. Invasive pneumococcal disease in the Australian Capital Territory and Queanbeyan region: Do high infant rates reflect more disease or better detection? J Paediatr Child Health. 2004; 40:184-8.

3. Lipsky BA, Boyko EJ, Inui TS, Koepsell TD. Risk factors for acquiring pneumococcal infections. Arch Intern Med. 1986; 146:2179-85.

4. Van Hoek AJ, Andrews N, Waight PA, Stowe J, Gates P, George $\mathrm{R}$, et al. The effect of underlying clinical conditions on the risk of developing invasive pneumococcal disease in England. J Infect. 65:17-24.

5. Song JY, Eun BW, Nahm MH. Diagnosis of Pneumococcal Pneumonia: Current Pitfalls and the Way Forward. Infection \& Chemotherapy. 2013; 45:351-66.

6. Reller LB, Weinstein MP, Werno AM, Murdoch DR. Laboratory Diagnosis of Invasive Pneumococcal Disease. Clinical Infectious Diseases. 2008; 46:926-32.

7. Huang SS, Johnson KM, Ray GT, Wroe P, Lieu TA, Moore MR, et al. Healthcare utilization and cost of pneumococcal disease in the United States. Vaccine. 2011; 29:3398-412.

8. Frieden T. Antibiotic resistance threats in the united States, 2013. United States centre for disease control and prevention 2013.

9. Al Musawi M. A retrospective epidemiological study of invasive pneumococcal infections in children aged $0-5$ years in Bahrain from 1 January 1999 to 31 December 2003. Vaccine. 2012; 30, Supplement 6:G2-G6.

10. Morrill HJ, Caffrey AR, Noh E, LaPlante KL. Epidemiology of pneumococcal disease in a national cohort of older adults. Infect Dis Ther. 2014; 3:19-33.

11. Kouchak F, Askarian M. Nosocomial Infections: The Definition Criteria. Iranian Journal of Medical Sciences. 2012; 37:72-3.

12. Hanna-Wakim R, Chehab H, Mahfouz I, Nassar F, Baroud M, Shehab $M$, et al. Epidemiologic characteristics, serotypes, and antimicrobial susceptibilities of invasive Streptococcus pneumoniae isolates in a nationwide surveillance study in Lebanon. Vaccine. 2012; 30 Suppl 6:G11-7.

13. Bechini A, Taddei C, Barchielli A, Levi M, Tiscione E, Santini MG, et al. A retrospective analysis of hospital discharge records for $\mathrm{S}$. pneumoniae diseases in the elderly population of Florence, Italy, 2010-2012. Hum Vaccin Immunother. 2015; 11:156-65.
14. Askim A, Mehl A, Paulsen J, DeWan AT, Vestrheim DF, Asvold $\mathrm{BO}$, et al. Epidemiology and outcome of sepsis in adult patients with Streptococcus pneumoniae infection in a Norwegian county 1993-2011: an observational study. BMC Infect Dis. 2016; 16:223.

15. Lexau CA, Lynfield R, Danila R, et al. Changing epidemiology of invasive pneumococcal disease among older adults in the era of pediatric pneumococcal conjugate vaccine. JAMA. 2005; 294:2043-51.

16. Lee CY, Chiu CH, Huang YC, Chung PW, Su LH, Wu TL, et al. Invasive pneumococcal infections: a clinical and microbiological analysis of 53 patients in Taiwan. Clinical Microbiology and Infection. 2003; 9:614-8.

17. Tsai H-Y, Lauderdale T-L, Wang J-T, Chen Y-S, Liu J-W, Huang $\mathrm{J}-\mathrm{H}$, et al. Updated antibiotic resistance and clinical spectrum of infections caused by Streptococcus pneumoniae in Taiwan: Emphasis on risk factors for penicillin nonsusceptibilities. Journal of Microbiology, Immunology and Infection. 2013; 46:345-51.

18. Al-Muhairi S, Zoubeidi T, Ellis M, Nicholls MG, Safa W, Joseph J. Demographics and microbiological profile of pneumonia in United Arab Emirates. Monaldi Arch Chest Dis. 2006; 65:13-8.

19. Al-Muhairi SS, Zoubeidi TA, Ellis ME, Safa WF, Joseph J. Risk factors predicting outcome in patients with pneumonia in AlAin, United Arab Emirates. Saudi Med J. 2006; 27:1044-8.

20. Mahboub B, Al Zaabi A, Al Ali OM, Ahmed R, Niederman MS, El-Bishbishi R. Real life management of community-acquired Pneumonia in adults in the Gulf region and comparison with practice guidelines: a prospective study. BMC Pulm Med. 2015; 15:112.

21. Kalin M, Örtqvist $\AA$, Almela M, Aufwerber E, Dwyer R, Henriques $B$, et al. Prospective Study of Prognostic Factors in CommunityAcquired Bacteremic Pneumococcal Disease in 5 Countries. The Journal of Infectious Diseases. 2000; 182:840-7.

22. Sherif S, Sumpio BE. Economic development and diabetes prevalence in MENA countries: Egypt and Saudi Arabia comparison. World J Diabetes. 2015; 6:304-11.

23. Federation. ID. The country report, Bahrain. 2015. In: the IDF Atlas [Internet]. Belgium: International Diabetes Federation. 7. Available from: http://reports.instantatlas.com/report/view/846 e76122b5f476fa6ef09471965aedd/BHR.

24. Federation. ID. The country report, United Arab Emirates. 2015. In: The IDF Atlas [Internet]. Belgium: International Diabetes Federation. 7. Available from: http://reports.instantatlas.com/ report/view/846e76122b5f476fa6ef09471965aedd/ARE.

25. Federation. ID. The country report, Italy. 2015. In: the IDF Atlas [Internet]. Belgium: International Diabetes Federation. 7. Available from: http://reports.instantatlas.com/report/view/846 e76122b5f476fa6ef09471965aedd/ITA. 
26. Trampuz A, Widmer AF, Fluckiger U, Haenggi M, Frei R, Zimmerli W. Changes in the Epidemiology of Pneumococcal Bacteremia in a Swiss University Hospital During a 15-Year Period, 19862000. Mayo Clinic Proceedings. 2004; 79:604-12.

27. Robinson KA, Baughman W, Rothrock G, et al. Epidemiology of invasive streptococcus pneumoniae infections in the united states, 1995-1998: Opportunities for prevention in the conjugate vaccine era. JAMA. 2001; 285:1729-35.

28. Ricketson LJ, Nettel-Aguirre A, Vanderkooi OG, Laupland KB, Kellner JD. Factors Influencing Early and Late Mortality in Adults with Invasive Pneumococcal Disease in Calgary, Canada: A Prospective Surveillance Study. PLoS ONE. 2013; 8:e71924.

29. Lääveri T, Nikoskelainen J, Meurman O, Eerola E, Kotilainen P. Bacteraemic Pneumococcal Disease in a Teaching Hospital in Finland. Scandinavian Journal of Infectious Diseases. 1996; 28:41-6.

30. Naidu K, Nabose I, Ram S, Viney K, Graham SM, Bissell K. A Descriptive Study of Nosocomial Infections in an Adult Intensive Care Unit in Fiji: 2011-12. J Trop Med. 2014; 2014:5.

Publish in The International Arabic Journal of Antimicrobial Agents

The Journal is an open access peer-reviewed journal that publishes scientific papers about all aspects of antimicrobials. The journal will publish original research articles, reviews, brief reports and case reports dealing with basic and clinical antibacterial agents, antiviral, antiprotozoals, antituberculuous, antifungal and antihelminthes agents. All manuscripts must be prepared in English, and are subject to a rigorous and fair peer-review process. Accepted papers will immediately appear online. The journal aims to advance the knowledge, attitude and the research of chemotherapy in the Arabic world in cooperation with international, national scientific and public societies as well as research centers with similar aims and objectives. 